\title{
Genetic structure and gene flow of the flea Xenopsylla cheopis in Madagascar and Mayotte
}

\author{
Mireille Harimalala ${ }^{1 *+}$, Sandra Telfer ${ }^{2+}$, Hélène Delatte ${ }^{3}$, Phillip C. Watts ${ }^{4}$, Adélaïde Miarinjara', \\ Tojo Rindra Ramihangihajason ${ }^{1}$, Soanandrasana Rahelinirina ${ }^{5}$, Minoarisoa Rajerison ${ }^{5}$ and Sébastien Boyer ${ }^{1}$
}

\begin{abstract}
Background: The flea Xenopsylla cheopis (Siphonaptera: Pulicidae) is a vector of plague. Despite this insect's medical importance, especially in Madagascar where plague is endemic, little is known about the organization of its natural populations. We undertook population genetic analyses (i) to determine the spatial genetic structure of $X$. cheopis in Madagascar and (ii) to determine the potential risk of plague introduction in the neighboring island of Mayotte.

Results: We genotyped 205 fleas from 12 sites using nine microsatellite markers. Madagascan populations of X. cheopis differed, with the mean number of alleles per locus per population ranging from 1.78 to 4.44 and with moderate to high levels of genetic differentiation between populations. Three distinct genetic clusters were identified, with different geographical distributions but with some apparent gene flow between both islands and within Malagasy regions. The approximate Bayesian computation (ABC) used to test the predominant direction of flea dispersal implied a recent population introduction from Mayotte to Madagascar, which was estimated to have occurred between 1993 and 2012. The impact of this flea introduction in terms of plague transmission in Madagascar is unclear, but the low level of flea exchange between the two islands seems to keep Mayotte free of plague for now.

Conclusion: This study highlights the occurrence of genetic structure among populations of the flea vector of plague, $X$. cheopis, in Madagascar and suggests that a flea population from Mayotte has been introduced to Madagascar recently. As plague has not been reported in Mayotte, this introduction is unlikely to present a major concern for plague transmission. Nonetheless, evidence of connectivity among flea populations in the two islands indicates a possibility for dispersal by fleas in the opposite direction and thus a risk of plague introduction to Mayotte.
\end{abstract}

Keywords: Madagascar, Mayotte, Xenopsylla cheopis, Microsatellites, Genetic structure, Gene flow, Plague

\section{Background}

The oriental rat flea, Xenopsylla cheopis (Siphonaptera: Pulicidae) is a holometabolous insect ectoparasite and was first described in Egypt [1], which is believed to represent its origin [2]. This species of flea is now cosmopolitan because of widespread dispersal (principally on ships) by its preferred rodent host, the black rat Rattus rattus (Rodentia: Muridae) [3]. Xenopsylla cheopis is also a frequent parasite on the brown rat $R$. norvegicus and can parasitize other small mammals [4, 5]. This flea

\footnotetext{
* Correspondence: hmireille@pasteur.mg

${ }^{\dagger}$ Equal contributors

${ }^{1}$ Medical Entomology Unit, Institut Pasteur of Madagascar, Ambatofotsikely,

PO box 1274, 101 Antananarivo, Madagascar

Full list of author information is available at the end of the article
}

draws particular attention because of its role as a vector of pathogens responsible for human diseases such as plague and murine typhus $[6,7]$. Indeed, it is thought to be the most efficient vector of the plague bacterium, Yersinia pestis [8] and can transmit the plague both between rodent hosts and to humans.

Plague is a re-emerging disease occurring in many regions of the World [9] but, more than $90 \%$ of worldwide cases in 2014-2015 were reported in Africa [9]; Madagascar being the most affected country [10]. Plague was introduced to the eastern coastal region of Madagascar in 1898 [11], apparently by ships from India [12] and subsequently, spread to other ports before reaching the Central Highlands in 1921, where it became 
endemic [13] inside the "plague focus" (altitude $>800 \mathrm{~m}$ ) [14]. Nevertheless, some areas outside this focus have epidemics, such as the District of Ikongo (altitude $\sim 750 \mathrm{~m})$ in $1998[15,16]$ and the District of Ambilobe (altitude $<500 \mathrm{~m}$ ) in 2011 [17]. Although apparently absent from coastal areas since the 1930s, plague reemerged in Mahajanga, a port in the north-west in 1991 with annual outbreaks between 1995 and 1999 [13]. Eighty-six per cent of suspected cases reported in Madagascar between 2007 and 2011 were classified as bubonic plague [14], reflecting the important role played by flea vectors in the transmission cycle.

In Madagascar, Rattus rattus, the principal plague reservoir is abundant and found in diverse habitats [18]. Two flea species (Pulicidae) are reported to be the main vectors of Y. pestis: Xenopsylla cheopis [19], and an endemic species Synopsyllus fonquerniei [20]. Xenopsylla cheopis can be found throughout most of Madagascar, independent of altitude [21], while S. fonquerniei is largely absent below $800 \mathrm{~m}$ [22]. Unlike $S$. fonquerniei, $X$. cheopis is predominantly found on $R$. rattus captured inside houses $[15,18,22]$ and is therefore thought to be an important vector for human cases. Population genetic and phylogeographic studies have provided important insights into the invasion history and population ecology of $R$. rattus and $Y$. pestis in Madagascar. A large nationwide study of $R$. rattus indicated the existence of only two clearly defined genetic groups corresponding to two separate introduction events, with one largely confined to the far north of the island and one reflecting a large spatial expansion of an introduction that occurred in the south [23]. In contrast, $Y$. pestis exhibited significant geographical separation among 15 identified subclades, implying largely local epidemiological cycles with limited gene flow [24]. However, the same study also found evidence of long distance transfers, probably humanmediated [24].

Despite the importance of $X$. cheopis for plague transmission in Madagascar, no studies of its population genetic structure have been conducted. Most studies of $X$. cheopis involve laboratory studies of the flea-bacteria relationship [8], the host-flea relationship [25] or insecticide treatment effects [26-28]. Field studies on population structure and dispersal in Madagascar and surrounding areas are required to improve our understanding of vector dynamics and the associated epidemiological risks.

For example, studies of natural populations of the plague flea vector Oropsylla hirsuta, which transmits plague bacterium to the black-tailed prairie dog, highlighted the lack of isolation by distance and spatial genetic structure of the flea, and demonstrated that recolonization of fleas from plague-free to plague zones occurred and caused a flea population expansion after epizootics [29]. The implication is that this flea has reasonable dispersal ability at this spatial scale, with the high estimated rate of gene flow exhibited by $O$. hirsuta potentially associated with dispersal by its hosts (prairie dogs and other mammals) [30].

Although the population genetic structure of parasites is often linked to the population genetic structure and dispersal of their hosts, the extent of this congruence will depend on the intimacy of the parasite-host association [31], and factors such as host specificity and time spent on host. In many cases, parasites are expected to show stronger structuring than their hosts due to limited dispersal abilities and lower effective population sizes [32]. A comparative study of the fur flea Listropsylla agrippinae and the nest flea Chiastopsylla rossi revealed different phylogeographic patterns, with the nest flea showing higher genetic divergence between sampling localities, presumably due to more restricted dispersal as a consequence of less time spent on hosts [33]. In other cases, a lack of concordance in the phylogeographic and population genetic structure between some flea species and their hosts suggests the potentially important role of dispersal by other sympatric hosts [30, 34]. As a fur flea, adult $X$. cheopis are predicted to have relatively frequent opportunities to be dispersed by their hosts. However, in Madagascar, unlike its principal host, X. cheopis is strongly geographically restricted to houses [22] and is therefore spatially restricted to a small subset of $R$. rattus populations. Other mammals that are less abundant than Rattus rattus, such as Mus musculus and Suncus murinus, can be infested with $X$. cheopis [22]. However, unlike $R$. rattus, these are peridomestic and largely restricted to around houses. Thus, we predict that X. cheopis populations will show much greater genetic structure than $R$. rattus populations due to a combination of limited dispersal and lower effective population sizes.

The dispersal of rodents and their fleas from the plague focus in Madagascar poses a serious potential health threat to other areas of Madagascar and to neighboring islands, such as Mayotte. Plague has not been reported in Mayotte, however, there is an important maritime trade route between Mayotte and north-west Madagascar. Our objectives were therefore: (i) to determine the genetic diversity and spatial genetic structure of $X$. cheopis populations in Madagascar; (ii) to determine the extent and pattern of any gene flow between $X$. cheopis populations in Madagascar and Mayotte, which may constitute an indication of plague introduction risk.

\section{Methods}

Specimen sampling

Samples of $X$. cheopis were collected in two areas of Mahajanga, Madagascar (the Port of Mahajanga and 
Marolaka) and the village of Longoni in Mayotte (Fig. 1). Additional specimens of $X$. cheopis were available from a further nine sites in Madagascar. All fleas were collected from small mammal hosts that had been trapped following published protocols [35]. Some specimens in Madagascar were collected as part of human plague outbreak responses and/or rodent-vector monitoring (Table 1). Fleas were collected alive on their hosts and preserved in $70 \%$ ethanol. Two hundred and five fleas were collected (Table 1) and species identification was performed by an expert taxonomist. When available, more than 10 specimens per site were analysed genetically.

\section{Isolation of microsatellite loci}

Genomic DNA was extracted using a DNeasy Blood and Tissue Kit (Qiagen, Hilden, Germany) from 25 X. cheopis fleas collected in Madagascar. Partial genomic libraries

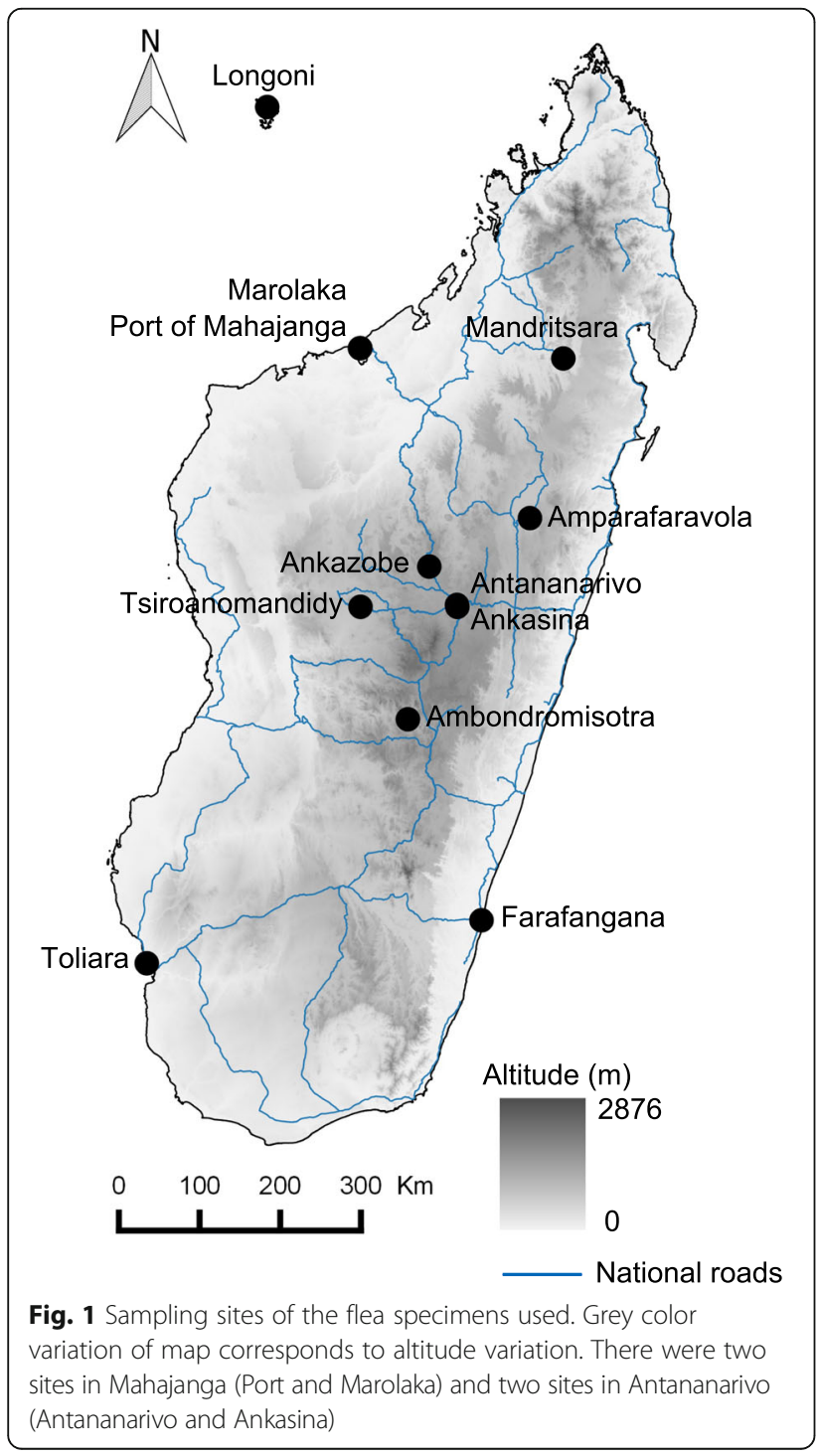

enriched for microsatellite repeats were constructed using the protocol described by $[36,37]$ and summarized in Additional file 1. After an initial screening of 44 candidate microsatellite loci for suitability for genotyping (Additional file 1), a panel of 12 loci were deemed suitable for genotyping based on ease of scoring (e.g. no spurious PCR products, few stutter bands) and apparent polymorphism (Table 2).

\section{DNA extraction, PCR and genotyping}

Genomic DNA for genotyping was extracted using the Instagene $^{\mathrm{Tm}}$ Matrix kit (Bio-Rad Laboratories Inc., California, USA) following the manufacturer's instructions. Multiplex PCR were performed by pooling microsatellite primers into three pools of 4 pairs of primers per pool (Table 2). Multiplex PCRs contained $7.5 \mu \mathrm{l}$ of Type-it microsatellite PCR kit $(2 \times)$ (Qiagen, Hilden, Germany), $0.4 \mu \mathrm{l}$ of each forward and reverse primers of the pool $(10 \mu \mathrm{M}), 1 \mu \mathrm{l}$ of DNA sample and sufficient water for a $15 \mu \mathrm{l}$ final reaction volume. Thermal amplification conditions were $95{ }^{\circ} \mathrm{C}$ for $5 \mathrm{~min}$, followed by 35 cycles of $95{ }^{\circ} \mathrm{C}$ for $30 \mathrm{~s}, 58{ }^{\circ} \mathrm{C}$ for $45 \mathrm{~s}, 72{ }^{\circ} \mathrm{C}$ for $55 \mathrm{~s}$ and a final extension step of $72{ }^{\circ} \mathrm{C}$ for $10 \mathrm{~min}$. PCR products were sized by capillary electrophoresis on an ABI prism 3130 (Applied Biosystems, California, USA) with GeneScan ${ }^{\mathrm{mi}} 500$ LIZ $^{\circ}$ Size Standard (Applied Biosystems, California, USA) and GENEMAPPER software (Applied Biosystems, California, USA).

\section{Statistical analysis}

Genotypic linkage disequilibrium between pairs of loci was tested with the exact test implemented in the GENEPOP v.4.3 [38]. The same software was used to estimate basic measures of genetic variability: mean number of alleles per locus $(\mathrm{Na})$, observed $(\mathrm{Ho})$ and expected (He) heterozygosities and $\mathrm{F}_{\mathrm{IS}}$ [39] for each population. Significance test for $\mathrm{F}_{\mathrm{IS}}$ values were performed using Hardy-Weinberg exact test implemented in GENEPOP. Allelic data were checked for null alleles, allelic dropout and stutter bands using MICROCHECKER v.2.2.3 with the Oosterhout algorithm [40]. Population differentiation $\left(\mathrm{F}_{\mathrm{ST}}\right)$ was estimated using ARLEQUIN v.3.5.2.1 [41]; the ENA method was used also to calculate an unbiased $\mathrm{F}_{\mathrm{ST}}\left(\mathrm{F}_{\mathrm{ST}}{ }^{\mathrm{ENA}}\right)$ using FREENA [42].

\section{Identification of genetic clusters}

We used two approaches to determine the number of distinct genetic populations: a Bayesian clustering approach using STRUCTURE [43] and the discriminant analysis of principal components (DAPC) [44].

STRUCTURE simultaneously identifies potential populations (clusters) and probabilistically assigns individuals to each of the $K$ populations based on the sample genotypes. STRUCTURE runs were performed using the 
Table 1 Sample sites, abundance of rats and fleas and variation in basic genetic diversity parameters

\begin{tabular}{|c|c|c|c|c|c|c|c|c|c|c|c|}
\hline Sites & Longitude & Latitude & $\begin{array}{l}\text { Year of } \\
\text { sampling }\end{array}$ & $\begin{array}{l}\text { Plague } \\
\text { epidemic }\end{array}$ & $\begin{array}{l}\text { No. of fleas } \\
\text { (No. of rodents) }\end{array}$ & Flea index ${ }^{a}$ & $\mathrm{Na}$ & $\mathrm{He}$ & $\mathrm{Ho}$ & $F_{I S}$ & $P$ \\
\hline \multicolumn{12}{|l|}{ Mayotte } \\
\hline Longoni & 45.159 & -12.733 & 2014 & No & $39(16)$ & 0.5 & 2.56 & 0.32 & 0.25 & $0.24^{*}$ & $<0.0001$ \\
\hline \multicolumn{12}{|l|}{ Madagascar } \\
\hline Marolaka & 46.314 & -15.725 & 2014 & No & $46(13)$ & na & 4.44 & 0.50 & 0.41 & $0.19^{*}$ & $<0.0001$ \\
\hline Port of Mahajanga & 46.309 & -15.725 & 2014 & No & $13(3)$ & 0.7 & 3.11 & 0.44 & 0.41 & 0.11 & 0.075 \\
\hline Ankasina & 47.512 & -18.910 & 2014 & Yes & $16(6)$ & 3.5 & 2.89 & 0.44 & 0.33 & $0.28^{*}$ & $<0.0001$ \\
\hline Amparafaravola & 48.416 & -17.833 & 2015 & Yes & $20(4)$ & 7.8 & 3.00 & 0.42 & 0.37 & $0.16^{*}$ & 0.008 \\
\hline Ankazobe & 47.169 & -18.434 & na & Yes & $21(7)$ & 0.7 & 3.11 & 0.44 & 0.30 & $0.34^{*}$ & $<0.0001$ \\
\hline Tsiroanomandidy & 46.316 & -18.933 & 2013 & Yes & 19 (na) & 5.4 & 2.22 & 0.25 & 0.27 & -0.06 & 0.770 \\
\hline Antananarivo & 47.516 & -18.933 & 1996 & na & 9 (na) & na & 2.43 & 0.33 & 0.07 & $0.79^{*}$ & $<0.0001$ \\
\hline Toliara & 43.666 & -23.350 & 2012 & No & $5(1)$ & 10.3 & 1.78 & 0.26 & 0.29 & 0 & 0.550 \\
\hline Farafangana & 47.816 & -22.816 & 2012 & No & $5(1)$ & 1.4 & 1.78 & 0.28 & 0.36 & -0.14 & 0.587 \\
\hline Mandritsara & 48.830 & $-15,852$ & 2014 & Yes & 7 (na) & 0.3 & 2.78 & 0.43 & 0.24 & $0.51^{*}$ & $<0.0001$ \\
\hline Ambondromisotra & 46.903 & -20.323 & 2013 & Yes & 5 (na) & 1.7 & 2.11 & 0.30 & 0.29 & 0.14 & 0.248 \\
\hline
\end{tabular}

Abbreviations: $N a$ average number of alleles per locus, He expected heterozygosity, Ho observed heterozygosity, $F_{I S}$ inbreeding coefficient, $P$ P-value for $F_{I S}$ na not available

${ }^{\text {a }}$ The flea index is the average number of fleas per rat. A flea index $>1$ represents a potential plague risk

*Significant $F_{I S}$ values; Hardy-Weinberg exact test: $P<0.05$

admixture model and correlated allele frequencies, with one million iterations of the Markov Monte Carlo Chain (MCMC) used as 'burn-in' that were followed by ten million MCMC iterations; the probability to observe the data $[\operatorname{Ln} \mathrm{P}(\mathrm{D})]$ was calculated for values of $\mathrm{K}$ ranging from 1 to 12, with five iterations for each $\mathrm{K}$-value. The best estimate of $K$ was taken to be the maximum value observed before the plateau of the curve Ln P(D) against K [43]. STRUCTURE HARVESTER [45] was also used to identify the most pronounced level of population structure using the method of Evanno et al. [46]. CLUMPP v.1.1.2 [47] was used to find the optimal alignment from replicate STRUCTURE runs, with the summary of results generated using DISTRUCT v.1.1 [48].

DAPC is a multivariate statistical method which uses the $k$-means clustering approach [44]. DAPC first transforms data using a principal components analysis (PCA) and subsequently identifies clusters using discriminant analysis (DA). This method thus defines genetic clusters, assigns individuals to clusters and allows visual assessment between-population differentiation. DAPC was implemented using the adegenet package [49] of the $\mathrm{R}$ software [50]. The identification of the number of genetic clusters was done using the function find.clusters with the prior that the maximum number of clusters is equal to $12(\mathrm{~K}=12)$.

\section{Inference of population introduction}

An approximate Bayesian computation $(\mathrm{ABC})$ analysis was conducted to infer the history of population introduction of $X$. cheopis between Mayotte and Madagascar. DIYABC v.2.0 [51] was used to test seven different scenarios of introduction history (Fig. 2). Three populations were chosen to test these scenarios: one from Mayotte (the port of Longoni) and two from Madagascar (Marolaka located in the coastal region of Mahajanga and Amparafaravola located in the Central Highlands; Fig. 1). Amparafaravola was chosen as the site to represent the Central Highlands as this sample was the most representative of the existing genetic variability. For each scenario and each population, the following demographic parameters were estimated: dates of founding of the different populations (as number of generations) (ti), current effective population size (as number of diploid individuals, $\mathrm{Ni}$ ), number of founders in the introduced populations (Nbi) and the duration of the initial bottleneck (dbi), which may be considered as a latency phase after each introduction event.

For all demographic parameters, prior distribution ranges were implemented according to current knowledge of $X$. cheopis. The generation time of $X$. cheopis varies between 18 days to 20 months according to biotic and abiotic conditions [52]. Laboratory conditions of $25 \pm 2{ }^{\circ} \mathrm{C}$ temperature and $70-80 \%$ relative humidity stimulate an average 12 generations per year. However, we note that natural populations of $X$. cheopis show seasonal dynamics in both the Central Highlands and Mahajanga [53]. Larval and pupal development times and survival can be affected by temperature and humidity [53] and so, generation times may be longer under 
Table 2 Summary of the microsatellite loci and PCR primers developed for the flea Xenopsylla cheopis

\begin{tabular}{|c|c|c|c|c|c|}
\hline Multiplex pool & Primer (locus) names & Primer sequences $\left(5^{\prime}-3^{\prime}\right)$ & Repeat motifs & Size range (bp) & Allele number \\
\hline \multirow[t]{8}{*}{ Pool 1} & XCO24 & F: 6-FAM-ATGCAGCTCGTTCGTCTCC & $C A(5) \ldots C A(13) \ldots C A(5)$ & ind & - \\
\hline & & R: GTCCAATTCATCCGCATCG & & & \\
\hline & XC009 & F: NED-CATTGCGGGAGCATCAG & $(C A) 5 \ldots(C A) 8$ & $283-303$ & 6 \\
\hline & & R: TGCAGGCACAAAATTCGAC & & & \\
\hline & XC018 & F: PET-TCGATTCAGCCGTTTTCG & (CA) 10 & ind & - \\
\hline & & R: TTGGAGAAGGAGATGTGTATGC & & & \\
\hline & XC037 & F: VIC-GGGCCACCGAGTTGACG & {$[(\mathrm{GTT}) 2 \ldots] 13$} & 290-348 & 4 \\
\hline & & R: TGGTGTTCCGTTACCGTTCC & & & \\
\hline \multirow[t]{8}{*}{ Pool 2} & XC007 & F: 6-FAM-CTGGTTGGATTGTCTCC & (CA) $5 \ldots(C A) 22$ & ind & - \\
\hline & & R: ACTATGCCGGATTAAGG & & & \\
\hline & XC021 & F: PET-AGTGGACCGAGAACAGAGC & (GT)9 & $242-258$ & 5 \\
\hline & & R: TCATGTAAAGAGACCTGAGACC & & & \\
\hline & SF009 & F: NED-CGTGTAGTTGCGAGAGAAGC & (GT)4...(GT)5 & $168-187$ & 5 \\
\hline & & R: GGAGAAGTGCGTTTACAGAGC & & & \\
\hline & XC013 & F: VIC-CAAAATTGGAGAAGGAGACG & [GT(2)..GTAT]5 & $164-235$ & 5 \\
\hline & & R: AAATCGTTGACGGAAGAAGC & & & \\
\hline \multirow[t]{8}{*}{ Pool 3} & XC023 & F: PET-CTAGTAAACGCAAACGCTACC & {$[G T(3) \ldots] 7$} & $300-380$ & 5 \\
\hline & & R: CCCCCAAACAAATCAGC & & & \\
\hline & XC044 & F: 6-FAM-AAAAGTAAAGTCGAACAAGTGG & (GTAT)6...(GT)5 ...(GTAT) 8 & $396-428$ & 5 \\
\hline & & R: GCTTATAGGTTACAAACATCTGG & & & \\
\hline & XC016 & F: NED-ATCGACCCCAAAATCAGC & GT(10)...GTAT(12)..GT(7) & $370-400$ & 11 \\
\hline & & R: ACCCCTGGTTGGATTGC & & & \\
\hline & XC002 & F: VIC-GCAGGCACAAAATTCGACA & GT(9)...GT(5) & 266-302 & 5 \\
\hline & & R: GCGGGGGCATCAGTTAAT & & & \\
\hline
\end{tabular}

Abbreviation: ind, pairs of primers tested did not provide specific pics in the targeted locus

field conditions. As these conditions are similar to those in our study sites, time (expressed in generations before sampling) was translated into years assuming 12 generations per year. One million simulations were conducted under each scenario (a total of 7 million generations). Posterior probabilities of each scenario were computed by performing direct approach and a polychotomous weighted logistic regression on the $1 \%$ of simulated datasets closest to the observed dataset $[54,55]$ after linear discriminant analysis on summary statistics [51]. Principal components analysis (PCA) was performed using summary statistics of simulated datasets and of observed dataset to check the suitability of the model (scenarios and prior parameters). Confidence in scenario choice was further tested using additional simulations. This included estimation of the probability of a type-II error (the probability of selecting the chosen scenario when it is not correct). After scenario choice, we proceeded to parameter inference estimated from the modes and $95 \%$ confidence intervals (CI) of their posterior distributions.
To determine directional relative migration between the three chosen populations, we used the divMigrate function from the R-package diveRsity v.1.9.89 [56] using Jost's D as measure of genetic distance [57] and with a bootstrap value of 1000. DivMigrate uses the method described in Sundqvist et al. [58].

\section{Results}

\section{Genetic variability}

Nine microsatellite markers produced PCR amplicons for all samples (XC009, XC037, XC021, SF009, XC013, XC023, XC044, XC016 and XC002; cf. Table 2) and only these nine loci were used in genetic analyses. The number of alleles at these 9 loci ranged from 4 to 11 (Table 2) and the mean number of alleles per locus and per populations $(\mathrm{Na})$ ranged from 1.78 to 4.44 (Table 1 ). The expected heterozygosity $(\mathrm{He})$ ranged from 0.25 to 0.50 while the observed heterozygosity $(\mathrm{Ho})$ varied from 0.07 up to 0.41 . All populations except those from Tsiroanomandidy, Toliara and Farafangana, showed a heterozygote deficit (i.e. $\mathrm{He}>\mathrm{Ho}$ and thus deviation 

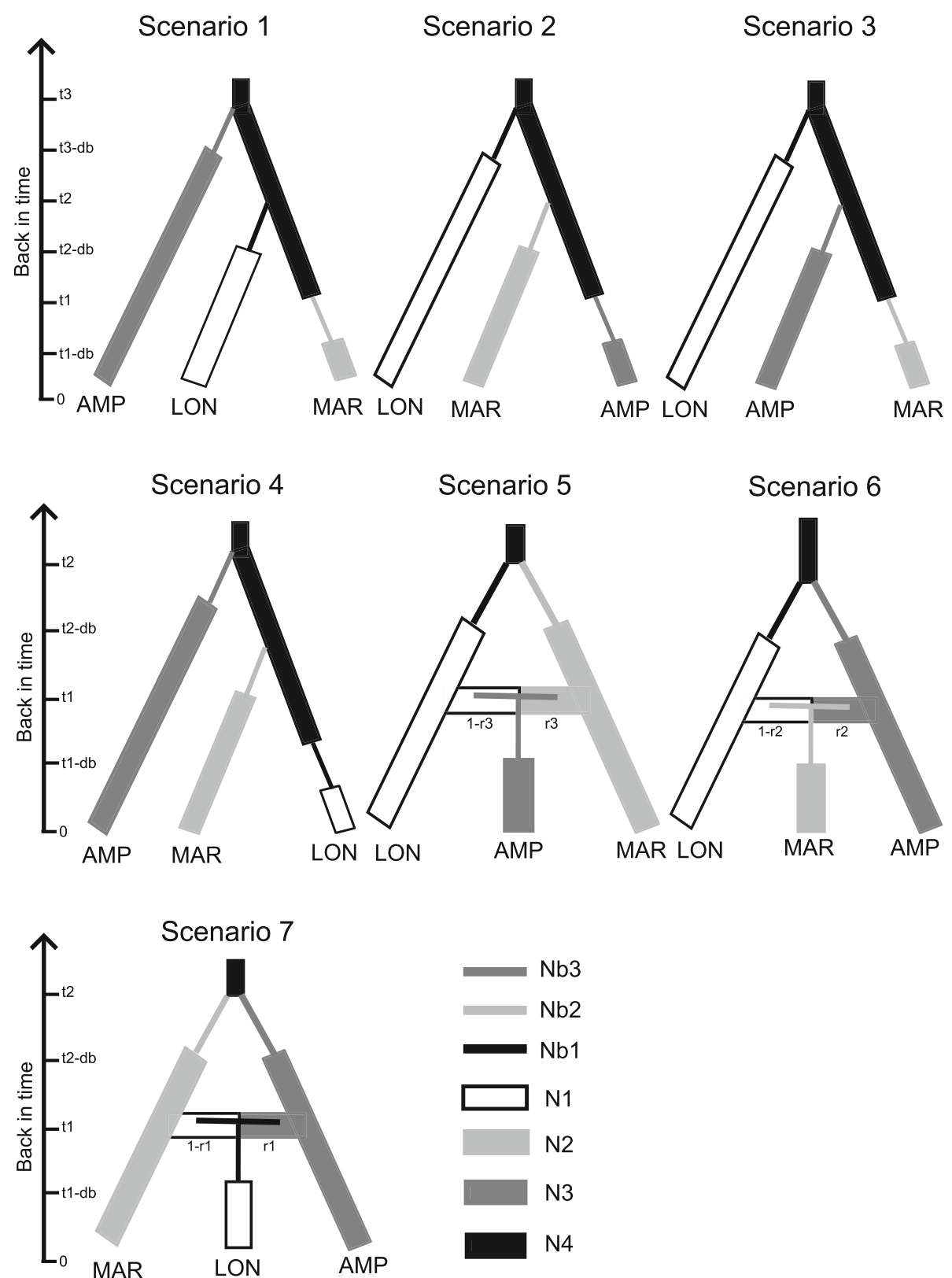

Fig. 2 Seven different scenarios inferred for the introduction history of Xenopsylla cheopis in Madagascar and Mayotte. Three populations were considered: population from Longoni in Mayotte (LON), population from Marolaka (MAR) and population from Amparafaravola (AMP) in Madagascar where N1, N2, N3 are their respective effective population sizes. Nb1, Nb2 and Nb3 correspond to the numbers of founders in the introduced population. Time scales corresponding to generations back in time from the sampling date (time 0 ) are shown at the left (t1, t2 and t3 generations ago); $d b$, is the duration of the initial bottleneck. For all the scenarios, LON, MAR and AMP derived from an unsampled ancestral population having N4 effective population size. Particularly for each of the scenarios 1-4, derivation from the ancestral population was independent. Scenario 1: AMP derived from the ancestral population at t3 followed by LON at t2 then, MAR at t1. Scenario 2: LON derived from ancestral population at $\mathrm{t} 3$ followed by MAR at $\mathrm{t} 2$ then, AMP at $\mathrm{t} 1$. Scenario 3: LON derived from ancestral population at $\mathrm{t} 3$ followed by AMP at $\mathrm{t} 2$ then, MAR at t1. Scenario 4: AMP derived from ancestral population at t 3 followed by MAR at t2 then, LON at t1. The remaining scenarios (5-7) assumed that two parental populations had diverged from an ancestral population at $\mathrm{t} 2$ before they would admix and gave the third population at t1. Scenario 5: the parental populations were LON and MAR and their admixture at a rate r3 gave AMP. Scenario 6: the parental populations were LON and AMP and their admixture at a rate 2 gave MAR. Scenario 7: the parental populations were MAR and AMP and their admixture at a rate $r 1$ gave LON 
from expected Hardy-Weinberg equilibrium conditions), and with 7 of the 12 sites having significantly positive $\mathrm{F}_{\mathrm{IS}}$ values (Hardy-Weinberg exact test; $P<0.05$ ) (Table 1). Only one of the 36 locus-pair combinations indicated significant linkage disequilibrium (XC002-XC009; $P<0.05)$. Estimated frequencies of null alleles per locus per population ranged from 0.16 to 0.45 ( 5 cases $\leq 0.20$; 9 cases between 0.20 and $0.30 ; 2$ cases between 0.30 and 0.40 ; and 1 case $>0.40$ ) (Additional file 2: Table S1).

\section{Population structure}

Both Bayesian clustering and multivariate methods identified three main genetic clusters: changes in Ln $\mathrm{P}(\mathrm{D})$ approached a plateau at $\mathrm{K}=4$ (Additional file 3: Figure $\mathrm{S} 1$ ), thus the best $\mathrm{K}$ value (the maximum $\mathrm{K}$ value observed before the plateau, [43]) was $\mathrm{K}=3$, the curve of DeltaK [46] showed a slope with a break occurring at $K=3$ and the graphical output yielded by DAPC also supported three genetic clusters (Fig. 3).

Genetic clusters had some underlying geographical structure, whereby all individuals from Mayotte were assigned to one cluster and individuals from Madagascar were assigned to three clusters including the cluster that contained samples from Mayotte (Fig. 4). All fleas from Mayotte had a membership coefficient $>90 \%$ to cluster 1. Fleas from some regions in Madagascar, namely Marolaka, the port of Mahajanga, Mandritsara and Antananarivo, also had substantial membership to cluster 1 as well as a second cluster (indicated in red in Fig. 4) that was only identified in Madagascar. Most individuals from Toliara and Farafangana had high membership coefficients to cluster 2, while fleas from
Ankasina and Ankazobe, had membership coefficients that indicated they belonged to cluster 2, but with more apparent genetic influence of the third cluster (indicated in yellow in Fig. 4). Fleas from Tsiroanomandidy and Ambondromisotra presented a membership coefficient greater than $90 \%$ to cluster 3 ; in Amparafaravola, cluster 3 was still dominant though some individuals appeared to belong to cluster 2 or cluster 1 (Fig. 4).

Division of populations into genetic groups was also reflected by the differentiation among these populations suggested by values of $\mathrm{F}_{\mathrm{ST}}$ (and $\mathrm{F}_{\mathrm{ST}}{ }^{\mathrm{ENA}}$; Additional file 4: Tables S2, S3). The $\mathrm{F}_{\mathrm{ST}}$ values varied from 0 (Tsiroanomandidy vs Ambondromisotra) to 0.51 (Antananarivo vs Ambondromisotra), with most values quite high (>0.25) and almost all values of $\mathrm{F}_{\mathrm{ST}}$ significantly different from 0 . The unbiased $\mathrm{F}_{\mathrm{ST}}{ }^{\mathrm{ENA}}$ values ranged from 0.01 (Port of Mahajanga vs Marolaka) to 0.45 (Antananarivo vs Ambondromisotra, Tsiroanomandidy $v s$ Longoni and Toliara vs Tsiroanomandidy) (Additional file 4: Tables S2, S3). Comparable with estimates of $\mathrm{F}_{\mathrm{ST}}$, most pairwise estimates of $\mathrm{F}_{\mathrm{ST}}{ }^{\mathrm{ENA}}$ were relatively high $(>0.25)$.

\section{Population introduction}

Using the direct approach, the highest posterior probability $(p)$ was obtained for scenario $6(p=0.50)$, with scenarios 7 and 5 having substantially lower probabilities ( $p=0.19$ and 0.18 respectively; Additional file 5 : Table $S 4)$; the logistic approach altered slightly the ranking of demographic scenarios, marginally favoring Scenario 6 $(P=0.48)$ and with Scenario 5 favored over $7(P=0.43$ and 0.06 , respectively). All other scenarios for both

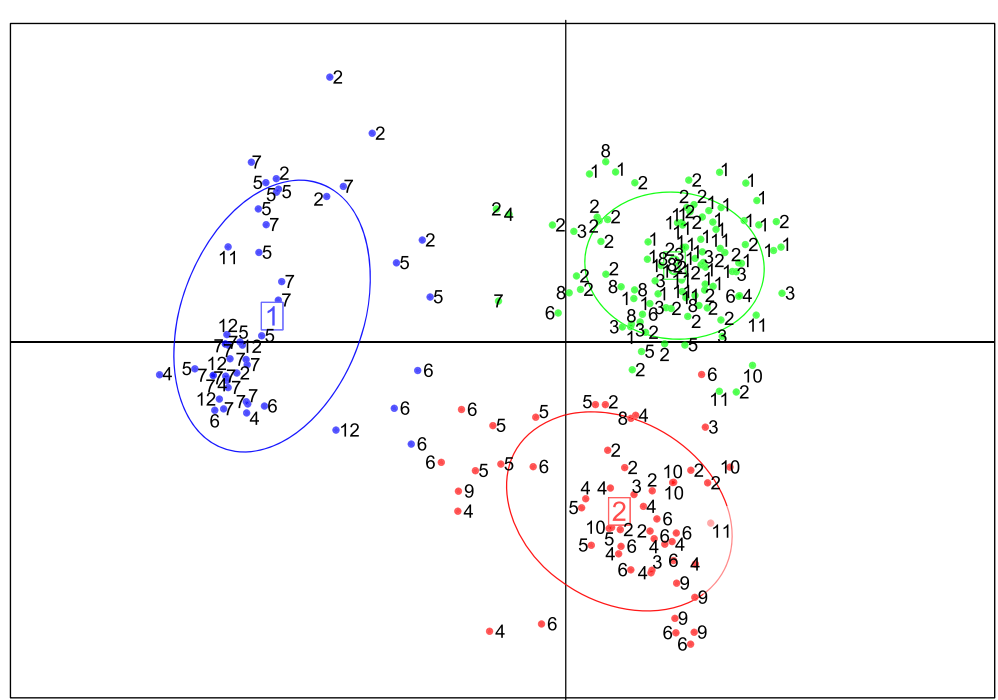

Fig. 3 Genetic clusters identified using the DAPC method. Individuals are shown by dots grouped into one of the three genetic clusters. Numbers quoted correspond to the sampling sites: 1, Longoni; 2, Marolaka; 3, Port of Mahajanga; 4, Ankasina; 5, Amparafaravola; 6, Ankazobe; 7, Tsiroanomandidy; 8, Antananarivo; 9, Toliara; 10, Farafangana; 11, Mandritsara; 12, Ambondromisotra 


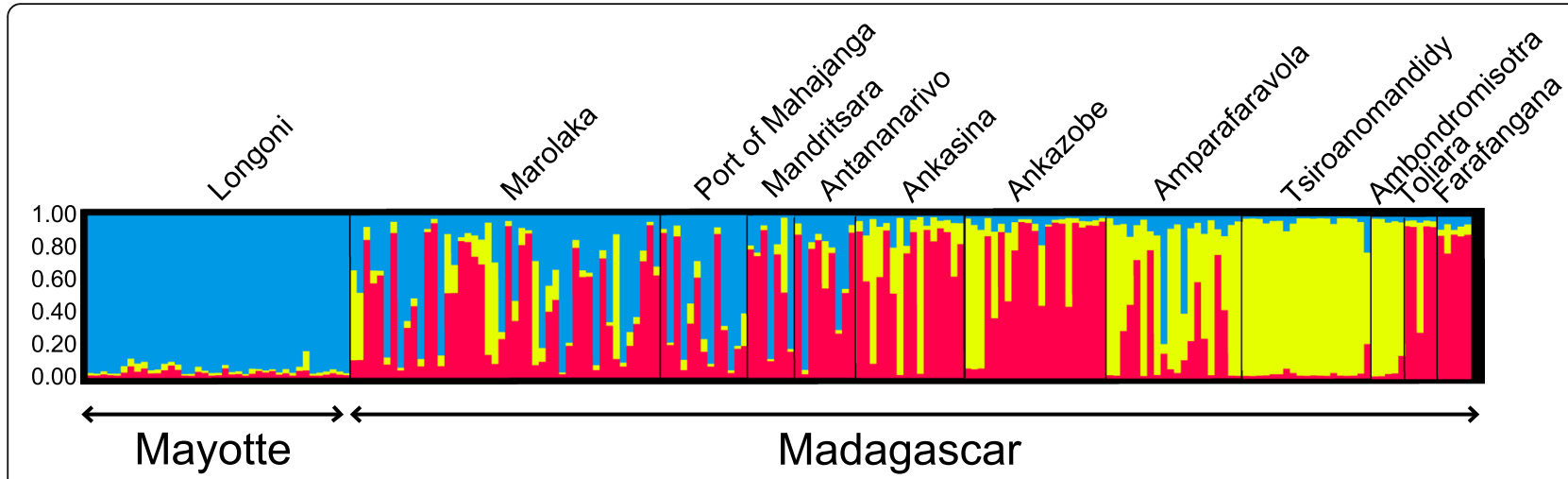

\section{Cluster 1}

Cluster 3

Fig. 4 Bar plot showing the genetic structure of individuals from Mayotte and Madagascar generated using STRUCTURE software. Three genetic clusters were assumed $(K=3)$ and are represented by three colors (blue, red and yellow clusters). Each vertical line represents an individual and the length of each colored line corresponds to the membership coefficient (scale at the left of the bar plot) for a cluster. Individuals are grouped according to their sampling sites

approaches had low $(<0.1)$ probabilities (Additional file 5: Table S4 and Additional file 6: Figure S2). The type II error rate (Additional file 7: Table S5) associated with Scenario 6 (probability to select Scenario 6 though it is not correct) was relatively low $(P=0.08)$ providing confidence in the scenario choice. The favored Scenario 6 assumes that the population from Marolaka was a result of an admixture of a population from Longoni and from Amparafaravola (Fig. 2). DivMigrate analysis supported Scenario 6 and suggested that asymmetric migrations occurred between flea populations of Mayotte and Madagascar. Flea population sources from Longoni (Mayotte) and Amparafaravola (Madagascar) migrated and constituted the sink population of Marolaka (Madagascar). High significant relative migration rates were obtained: 0.87 (Longoni to Marolaka) and 1 (Amparafaravola to Marolaka) (Additional file 8: Table S6).

Estimates for the different parameters inferred using scenario 6 are given in Table 3. It was estimated that about 47 generations had occurred (95\% CI: 16.4-241) between the time of sampling ( $\mathrm{t} 0)$ and the time of population admixture (t1) (Fig. 2; Scenario 6). Assuming 12 generations per year for $X$. cheopis and that the samples were collected in 2014, the population from Marolaka is estimated to have been affected by an introduction that occurred between 1993 and 2012 (t1: 2010 [95\% CI: 1993-2012]) at an admixture rate of $\mathrm{r} 2=55.8 \%$ (95\% CI: $21-82 \%$ ) (Table 3). If fewer generations per year occur under field conditions, a date towards the beginning of this time period is more likely.

\section{Discussion}

Plague is a health problem in Madagascar and there is a need to understand the natural organization and dynamics of the key vector, Xenopsylla cheopis. This first population genetic analysis of $X$. cheopis from Madagascar and Mayotte makes important advances in our understanding: Xenopsylla cheopis populations are genetically and geographically structured in Madagascar, with interesting differences compared to previous studies of the principal host $R$. rattus and, a recent population migration of this flea occurred from Mayotte to Madagascar.

\section{Spatial genetic structure of fleas in Madagascar}

Within Madagascar, although there was fairly high genetic differentiation between populations, there was evidence of some gene flow between populations. As predicted, $X$. cheopis appears to show stronger population genetic structure than rats, with three clusters present in Madagascar compared to two for rats [23], and higher $\mathrm{F}_{\mathrm{ST}}$ values (most values $>0.25$ ), compared with a range of $0.01-0.21$ for rats [23]. Although these patterns may suggest more limited dispersal in the parasite compared to the host, other factors such as lower effective population size may also play a role [31, 32]. Moreover, as several populations showed shared membership of different genetic clusters and the bestsupported scenarios from the $\mathrm{ABC}$ analysis (Scenarios 6, 7 and 5) assume population admixture, whilst flea populations are genetically structured, there is also clear evidence of gene flow among populations.

Thus, comparisons of our results for $X$. cheopis with the results for $R$. rattus reveal some similarities and some interesting differences. It is notable that the $R$. rattus genetic structure appears to broadly follow a north/ south divide [23]. The northern genetic type of rats was largely restricted to the far north, with STRUCTURE 
Table 3 Summary of the DIYABC analysis based on 999,083 simulated datasets averaged over 9990 selected datasets

\begin{tabular}{|c|c|c|c|c|c|}
\hline Parameter names & Prior range $^{a}$ & Posterior parameter estimates ${ }^{a}$ & $95 \% \mathrm{Cl}^{\mathrm{a}}$ & Relative bias ${ }^{a}$ & Relative square root error ${ }^{\mathrm{a}}$ \\
\hline \multicolumn{6}{|c|}{ Effective population size } \\
\hline$N_{\text {IMay. }}$ & $10-2000$ & 377 & $113-1820$ & 0.14 & 0.36 \\
\hline$N_{2 \text { Mad-Maro. }}$ & $500-15,000$ & 586 & $654-11,300$ & -0.65 & 0.74 \\
\hline$N_{3 \text { Mad-Ampa }}$ & $200-4000$ & 644 & $394-3720$ & -0.40 & 0.47 \\
\hline$N_{4 T G h o s t}$ & $100-40,000$ & 3080 & $2230-23,800$ & 0.44 & 0.59 \\
\hline$N_{b 1}$ & $10-2000$ & 673 & $276-1770$ & 0.11 & 0.24 \\
\hline$N_{b 2}$ & $10-2000$ & 1660 & 674-1930 & 0.13 & 0.27 \\
\hline$N_{b 3}$ & $10-2000$ & 1030 & $373-1830$ & 0.14 & 0.27 \\
\hline \multicolumn{6}{|l|}{ Time in generation } \\
\hline$t_{1}$ & $2-1000$ & 46.6 & $16.4-241$ & 0.17 & 0.31 \\
\hline$t_{2}$ & $2-1500$ & 190.0 & $94.8-1220$ & 0.20 & 0.31 \\
\hline$d_{b}$ & $10-2000$ & 360 & $113-1860$ & 0.57 & 0.76 \\
\hline \multicolumn{6}{|c|}{ Genetic parameters (rate) } \\
\hline$\mu_{\text {mic }}$ & $1.00 \times 10^{-5}-1.00 \times 10^{-3}$ & $1.00 \times 10^{-4}$ & $1.00 \times 10^{-4}-2 \times 10^{-4}$ & -0.09 & 0.30 \\
\hline$p_{\text {mic }}$ & $1.00 \times 10^{-1}-9.00 \times 10^{-1}$ & $9.00 \times 10^{-1}$ & $5.05 \times 10^{-1}-9.00 \times 10^{-1}$ & 0.13 & 0.53 \\
\hline$s n_{\text {mic }}$ & $1.00 \times 10^{-8}-1.00 \times 10^{-5}$ & $1.00 \times 10^{-8}$ & $1.15 \times 10^{-8}-3.39 \times 10^{-6}$ & -0.99 & 0.99 \\
\hline$r 2$ & $0.001-0.999$ & 0.558 & $0.210-0.817$ & -0.022 & 0.37 \\
\hline
\end{tabular}

${ }^{a}$ Calculated for the best scenario (scenario 6)

analysis revealing only three sites out of 35 with evidence of mixed ancestry for some individuals. These three sites were geographically located between the northerly and southerly sites and included Mahajanga and a site close to Mandritsara, both sites in our study of $X$. cheopis which showed shared membership of different clusters. Although our results therefore suggest some congruence with the phylogeographic structure of hosts, as suggested for another fur flea, Listropsylla agrippinae, in South Africa [33], in general gene flow in $X$. cheopis did not appear to be strongly associated with geographical distance and $R$. rattus genetic structure. Specifically, some sites further south also showed shared membership of different clusters (Antananarivo, Amparafaravola), whilst, unlike for $R$. rattus [23], different flea populations within the Central Highlands could differ substantially in their proportion of membership to different clusters (e.g. Ankazobe vs Tsiroanomandidy and Ambondromisotra), indicating that in some cases little gene flow occurs between populations separated by relatively short distances. These apparently contradictory results could be explained by differences in the relative importance of human-mediated dispersal between $R$. rattus and $X$. cheopis and between sites.

A number of factors may lead to more frequent successful dispersal of fleas than black rats. Individual rats may carry a large number of fleas (in a study of rural highland villages in Madagascar, the average number of fleas on rats in houses was $>2$ [18]). Flea eggs, larvae and pupae are found in dust and debris from activities such as rice pounding [5], suggesting stages other than adults may also be potentially dispersed by human activity. Moreover, the competitive advantage of residents relative to migrants may be more significant for rats than fleas. Critically though, in urban sites that are linked by major roads and a high frequency of human movements, and are therefore more likely to experience human-mediated dispersal, $R$. rattus have largely been replaced by $R$. norvegicus (e.g. Mahajanga and Antananarivo [22]). Thus, at least in the last 30-40 years [22], human-mediated dispersal of $X$. cheopis may be much higher than for $R$. rattus. In contrast, dispersal of $R$. rattus and fleas in more remote, rural sites may be dominated by non-human mediated short-distance dispersal events. As $R$. rattus is abundant and widespread, occurring in diverse habitats [18], this would favor cumulative high levels of gene flow across rural landscapes. Indeed, in a population genetic study of rats at a more local landscape scale, rat populations showed only weak genetic differentiation between adjacent villages, with genetic structure at least partly related to topographic relief [59]. In contrast, the restriction of $X$. cheopis to rats and other peridomestic mammals living inside houses is likely to mean that successful, non-human mediated dispersal between villages is relatively rare.

Whilst fleas did show more genetic structuring than rats, given the patchy distribution of $X$. cheopis in rural landscapes and the likely effects on dispersal rates and 
effective population sizes, the number of clusters detected in Madagascar (three) is perhaps surprisingly low. This is especially true when compared to the more significant, local clustering of $Y$. pestis [24], despite the more widespread distribution of $Y$. pestis in the landscape (transmitted between rats in habitats other than houses by the endemic flea S. fonquerniei) and presumably increased opportunities for dispersal. Although our results do suggest significant levels of gene flow, likely via human-mediated dispersal, they may also reflect the restricted nature of our study as sampling too few individuals per site can lead to underestimation of the number of clusters [60] and incorrect cluster assignment [61]. However, simulation studies have indicated that reasonably limited sampling (6-10 individuals per site) can detect cryptic population structure and that any effects of low sample size are less with hierarchical population structure [60]. In our study, some sites in the south had low sample size from few hosts (Ambondromisotra, Toliara and Farafangana). Despite this, whilst Ambondromisotra had high membership coefficient to cluster 3 and Farafangana to cluster 2, Toliara showed a mix of individuals with high membership coefficient to clusters 2 and 3 .

Other factors may also influence the observed patterns of genetic variation within and between populations. Nine among twelve populations of $X$. cheopis showed heterozygote deficit which is significant in almost all cases. Evolutionary processes such as genetic drift, natural selection, inbreeding, mutation, population bottleneck or gene flow may influence allele frequencies and cause heterozygote deficit. Several of these may have contributed to our results. In addition to the gene admixture and gene flow between populations discussed above, fleas obtained from individual rats or rat families may be related, whilst population bottlenecks could occur within the spatially restricted populations of $X$. cheopis or due to seasonal dynamics in highland populations [53]. Increasing the number of sites at both the national scale and landscape scale, and standardizing the sample size within sites, would undoubtedly clarify the population structure of $X$. cheopis within Madagascar and elucidate the role of rat dispersal and human-mediated dispersal. However, our rather limited study already yields important insights, possibly due to the strong hierarchical structure inherent within parasite populations.

\section{Flea exchange between Mayotte and Madagascar}

Our results indicate that limited flea exchange does occur between Mayotte and north Madagascar, with both the $\mathrm{ABC}$ analysis and the divMigrate analyses suggesting asymmetric gene flow from Mayotte to Madagascar. This exchange is likely to be linked to commerce and shipping routes in the Indian Ocean and reflects similar patterns in previous studies of other species. The mitochondrial haplotype group associated with the $R$. rattus introduction to north Madagascar [23] has previously been found in East Africa and Grande Comore, whilst the mitochondrial haplotypes associated with the southern $R$. rattus introduction was found in Mayotte [62]. This led to the conclusion that Mayotte was colonized by $R$. rattus from Madagascar [62]. Interestingly, although we find evidence of a relatively recent successful introduction of $X$. cheopis from Mayotte, only one $R$. rattus individual in Madagascar has been shown to carry a mitochondrial haplotype related to European human colonization [62], again possibly reflecting a greater success of flea migrants compared to rat migrants as discussed above. Successful introduction is conditioned by the introduction of some rats carrying fleas that are able to successfully reproduce.

\section{Relevance of findings for plague introduction and dynamics}

Our findings are important for assessing the epidemiological risk of plague introduction in plague free areas such as Mayotte. Mayotte already supports populations of both a plague-competent vector species (X. cheopis) and plague-competent mammal host species such as $R$. rattus and an insectivore found to be infected with $Y$. pestis in Mahajanga (Suncus murinus) [22]. Although port cities such as Mahajanga or Longoni are hubs allowing plague introduction and intercontinental spread [63], the infrequent movement of fleas (and presumably their hosts) between the two ports indicated by this study suggest that the risk is real but limited. As there have been no plague outbreaks in humans in Mahajanga after the outbreaks in 1990s [14] and genetic studies of $Y$. pestis indicate that Mahajanga outbreaks were triggered by dispersal of infected rodents or fleas from the Central Highlands [63], the risk may have been further reduced because plague is no longer circulating in this region. However, studies of the peridomestic mammal and flea communities in Mahajanga are needed to assess this.

Even if plague did arrive in Mayotte due to the movement of infected fleas or hosts, many other factors would influence plague establishment and the risk of epidemics in the human population. One such factor is vector competence. Although the genetic differentiation among populations of $X$. cheopis is not associated with morphological differences, genetic differences might affect vector competence or resistance to pathogens [64] and therefore impact on disease transmission. Correlation between genetic structure of the insect vector and heterogeneity of vector competence has been reported for other disease-vector systems, for example Aedes albopictus (insect vector of dengue and yellow fever viruses) [65-67]. Interestingly, 
Tsiroanomandidy and Ambondromisotra, where cluster 3 occurs, are 'plague focus' areas where outbreaks occur every year. More work would need to be conducted to determine if different natural populations of $X$. cheopis differ in their vector competence.

Clearly, to further understand the risk of plague introduction and establishment in neighboring countries such as Mayotte as well as plague outbreaks in Madagascar outside the plague focus, there is a need for further, more extensive studies of flea and rat dynamics and dispersal, including for the endemic vector species, S. fonquerniei.

\section{Conclusion}

This study shows strong spatial structure among populations of the flea vector of plague, $X$. cheopis, from Madagascar and the nearby island of Mayotte. Gene flow occurred between Madagascar and Mayotte, but with evidence for a flea population having been introduced recently from Mayotte to Madagascar. As X. cheopis is the main vector of plague in Madagascar, the introduction of individuals to Mayotte may present a risk of plague introduction to this island.

\section{Additional files}

Additional file 1: Protocol describing microsatellite primers development (partial genomic libraries enrichment). (DOCX $19 \mathrm{~kb}$ )

Additional file 2: Table S1. Estimated frequencies of null alleles per locus per population using Van Oosterhout's method. (XLSX $10 \mathrm{~kb}$ )

Additional file 3: Figure S1. Identification of the number of genetic clusters using the methods of Pritchard \& Evanno $[43,46]$. (a) Posterior probability $L(K)$ and (b) DeltaK obtained based on $K$ numbers of genetic populations ranging from 1 to 10 . (PDF $52 \mathrm{~kb}$ )

Additional file 4: Table S2. Pairwise $F_{\mathrm{ST}}$ and values between all populations. Table S3. Pairwise $F_{\mathrm{ST}}{ }^{\mathrm{ENA}}$ values among populations. (XLSX $12 \mathrm{~kb}$ )

Additional file 5: Table S4. Probability for each of the seven tested scenarios of population introduction using direct and logistic approaches. (XLSX $9 \mathrm{~kb})$

Additional file 6: Figure S2. Raw results obtained for the seven scenarios tested using DIYABC software. (a) Posterior probabilities of scenarios obtained through a logistic regression computed every $10 \%$ (between 10 and 100\%) of the number of selected datasets. (b) PCA plot allowing to visualize how close datasets simulated (each small dot) under each scenario (different colors) are from the observed dataset (large yellow dot). The most relevant scenario chosen was scenario 6 . The probabilities for each scenario using direct and logistic approaches are given. (PDF $786 \mathrm{~kb}$ )

Additional file 7: Table S5. Confidence in scenario choice (using posterior based error computations). Pseudo-observed datasets (pods) were drawn from 500 simulated datasets closest to the observed dataset $(s=500)$. (DOCX $17 \mathrm{~kb})$

Additional file 8: Table S6. Bidirectional estimate of relative migration rates. The data give relative migration values by pairwise comparisons of the three populations from Madagascar and Mayotte. Analysis was performed using the function divMigrate from the package diveRsity [56]. Jost's D statistic was used to measure the genetic distance [57] and the bootstrap replicates were set to 1000. (XLSX $9 \mathrm{~kb}$ )

\section{Abbreviations}

ABC: Approximate Bayesian computation; DAPC: Discriminant analysis of principal components; ENA: Excluding Null Allele; MCMC: Markov Monte Carlo Chain; PCA: Principal components analysis

\section{Acknowledgements}

Thanks to the GIS team of the Institut Pasteur of Madagascar (IPM) for supplying base maps. Also, thanks to the IBIZA platform of the Mixed Research Unit (UMR PVBMT) of CIRAD in La Réunion for hosting genotyping lab works. Many thanks for Christophe Simiand for his help during genotyping lab-works.

\section{Funding}

Epidemiological surveys and laboratory works were funded by the Agence Régional de la Santé - Océan Indien (ARS-OI) (n²/DSP/Etudes et Statistiques/ 2013) and the Institut Pasteur of Madagascar. Microsatellite primer development was supported by the Wellcome Trust (a part of Research Career Development Fellowship to S. Telfer \#081705 and Senior Fellowship to S. Telfer \#095171).

\section{Availability of data and materials}

The microsatellite datasets supporting the conclusions of this article are available in the GenBank repository (see Table 1 for accession numbers).

\section{Authors' contributions}

TRR, SR and MR carried out flea samplings. TRR performed morphological identification of specimens. ST and PCW developed microsatellite loci. MH, TRR and AM extracted DNA from flea specimens. MH and HD set up multiplex PCR conditions for microsatellite markers. $\mathrm{MH}$ and $\mathrm{HD}$ performed genotyping and data analysis. MH, HD and ST wrote the paper. MH, ST, PCW, $A M, M R$ and SB proofread the paper. MR and SB managed the project and the team. All authors read and approved the final manuscript.

Ethics approval and consent to participate

Not applicable.

\section{Consent for publication}

Not applicable.

\section{Competing interests}

The authors declare that they have no competing interests.

\section{Publisher's Note}

Springer Nature remains neutral with regard to jurisdictional claims in published maps and institutional affiliations.

\section{Author details}

${ }^{1}$ Medical Entomology Unit, Institut Pasteur of Madagascar, Ambatofotsikely, PO box 1274, 101 Antananarivo, Madagascar. ${ }^{2}$ School of Biological Sciences, University of Aberdeen, Aberdeen AB24 2TZ, UK. ${ }^{3}$ UMR PVBMT, CIRAD, 7 Chemin de I'IRAT, Saint Pierre, La Réunion, France. ${ }^{4}$ Department of Ecology and Genetics, University of Oulu, Fl-90014 Oulu, Finland. ${ }^{5}$ Plague Unit, Institut Pasteur of Madagascar, Ambatofotsikely, PO box 1274, 101 Antananarivo, Madagascar.

Received: 10 January 2017 Accepted: 13 July 2017

Published online: 20 July 2017

\section{References}

1. Rothschild NC. New species of Siphonaptera from Egypt and the Soudan. Entomol Mon Mag. 1903;39:83-7.

2. Gratz N. Rodent reservoirs and flea vectors of natural foci of plaque. In: WHO Division of communicable diseases, editors. Plague manual: epidemiology, distribution, surveillance and control. Geneva, Switzerland: World Health Organization; 1999. p. 63-96.

3. Smit FGAM. The fleas of New Zealand (Siphonaptera). J Roy Soc New Zeal. 1979:9(2):143-232.

4. Beaucournu J-C, Fontenille D. Contribution à un catalogue des puces de Madagascar (Insecta, Siphonaptera). Arch Inst Pasteur Madagascar. 1991;59:57-98. 
5. Klein J, Uilenberg G. Données faunistiques et écologiques sur les puces de Madagascar (Siphonaptera). Cah ORSTOM Sér Entomol Méd Parasitol. 1966;4(8):31-60.

6. Bitam I, Dittmar K, Parola P, Whiting MF, Raoult D. Fleas and flea-borne diseases. Int J of Infect Dis. 2010;14:e667-e76.

7. Eisen RJ, Gage KL. Transmission of flea-borne zoonotic agents. Annu Rev Entomol. 2012;57:61-82.

8. Eisen RJ, Wilder AP, Bearden SW, Montenieri JA, Gage KL. Early-phase transmission of Yersinia pestis by unblocked Xenopsylla cheopis (Siphonaptera: Pulicidae) is as efficient as transmission by blocked fleas. J Med Entomol. 2007:44:678-82.

9. Bertherat EG. Plague in Madagascar: overview of the 2014-2015 epidemic season. Week Epidemiol Rec. 2015;90(20):250-2.

10. Bertherat EG. Plague around the world, 2010-2015. Week Epidemiol Rec. 2016:91(8):89-93.

11. Brygoo ER. Epidemiologie de la peste à Madagascar. Arch Inst Pasteur Madagascar. 1966:35:9-147.

12. Morelli G, Song Y, Mazzoni CJ, Eppinger M, Roumagnac P, Wagner DM, et al. Yersinia pestis genome sequencing identifies patterns of global phylogenetic diversity. Nat Genet. 2010;42:1140-3.

13. Chanteau S, Ratsitorahina M, Rahalison L, Rasoamanana B, Chan F, Boisier P, et al. Current epidemiology of human plague in Madagascar. Microbes Infect. 2000;2(1):25-31.

14. Andrianaivoarimanana V, Kreppel K, Elissa N, Duplantier JM, Carniel E, Rajerison M, Jambou R. Understanding the persistence of plague foci in Madagascar. PLoS Negl Trop Dis. 2013;7:1-8.

15. Duplantier J-M, Duchemin JB, Ratsitorahina M, Rahalison L, Chanteau S. Résurgence de la peste dans le district d'lkongo à Madagascar en 1998. 2. Réservoirs et vecteurs impliqués. Bull Soc Pathol Exot. 2001:94(2):119-22.

16. Migliani R, Ratsitorahina M, Rahalison L, Rakotoarivony I, Duchemin JB, Duplantier JM, et al. Résurgence de la peste dans le District d'lkongo à Madagascar en 1998. 1. Aspects épidémiologiques dans la population humaine. Bull Soc Pathol Exot. 2001;94(2):115-8.

17. Richard V, Riehm JM, Herindrainy P, Soanandrasana R, Ratsitorahina M Rakotomanana F, et al. Pneumonic plague outbreak, northern Madagascar, 2011. Emerg Infect Dis. 2015;21:8-15.

18. Rahelinirina S, Duplantier JM, Ratovonjato J, Ramilijaona O, Ratsimba M, Rahalison L. Study on the movement of Rattus rattus and evaluation of the plague dispersion in Madagascar. Vector Borne Zoonotic Dis. 2010;10(1):77-84.

19. Beaucournu J-C. Diversité des puces vectrices en fonction des foyers pesteux. Bull Soc Pathol Exot. 1999;92(5 Bis):419-21.

20. Brygoo ER, Dodin A. Transmission expérimentale de la peste par Synopsyllus fonquerniei de Madagascar. C R hebdomadaires Sci Acad Sci Série D: Sci Nat. 1967;264(4):681-2.

21. Duplantier J-M, Rakotondravony D. The rodent problem in Madagascar: agricultural pest and threat to human health. In: Singleton GR, Hinds LA Leirs H, Zhang Z, editors. Ecologically-based rodent management. Canberra: ACIAR publications; 1999. p. 441-59.

22. Duplantier J-M, Duchemin J-B, Chanteau S, Carniel E. From the recent lessons of the Malagasy foci towards a global understanding of the factors involved in plague reemergence. Vet Res. 2005;36:437-53.

23. Brouat C, Tollenaere C, Estoup A, Loiseau A, Sommer S, Soanandrasana R, et al. Invasion genetics of a human commensal rodent: the black rat Rattus rattus in Madagascar. Mol Ecol. 2014;23:4153-67.

24. Vogler AJ, Chan F, Wagner DM, Roumagnac P, Lee J, Nera R, et al. Phylogeography and molecular epidemiology of Yersinia pestis in Madagascar. PLoS Negl Trop Dis. 2011:5(9):e1319.

25. Mears S, Clark F, Greenwood M, Larsen K. Host location, survival and fecundity of the oriental rat flea Xenopsylla cheopis (Siphonaptera: Pulicidae) in relation to black rat Rattus rattus (Rodentia: Muridae) host. Bull Entomol Res. 2002:92:375-84

26. Boyer S, Miarinjara A, Elissa N. Xenopsylla cheopis (Siphonaptera: Pulicidae) susceptibility to deltamethrin in Madagascar. PLoS One. 2014;9(11):e111998.

27. Ratovonjato J, Duchemin JB, Duplantier J-M, Chanteau S. Xenopsylla cheopis (Siphonaptera: Xenopsyllinae), puces des foyers ruraux de peste des Hautes Terres malgaches: niveau de sensibilité au DDT, aux pyréthrinoïdes et aux carbamates après 50 années de lutte chimique. Arch Inst Pasteur Madagascar. 2000;66:9-12.

28. Zhang Y-C, Qi Y-M. Histological effects of fenoxycarb on the oriental rat flea, Xenopsylla cheopis (Siphonaptera: Pulicidae). Acta Entomol Sin. 2008;51(5):504-8.
29. Brinkerhoff RJ, Martin AP, Jones RT, Collinge SK. Population genetic structure of the prairie dog flea and plague vector, Oropsylla hirsuta. Parasitology. 2011;138(1):71-9.

30. Jones PH, Britten HB. The absence of concordant population genetic structure in the black-tailed prairie dog and the flea, Oropsylla hirsuta, with implications for the spread of Yersinia pestis. Mol Ecol. 2010;19:2038-49.

31. Huyse T, Poulin R, Theron A. Speciation in parasites: a population genetics approach. Trends Parasitol. 2005;21(10):469-75.

32. du Toit N, van Vuuren BJ, Matthee S, Matthee CA. Biogeography and host related factors trump parasite life history: limited congruence among the genetic structures of specific ectoparasitic lice and their rodent hosts. Mol Ecol. 2013;22(20):5185-204.

33. van der Mescht L, Matthee S, Matthee C. Comparative phylogeography between two generalist flea species reveal a complex interaction between parasite life history and host vicariance: parasite-host association matters. BMC Evol Biol. 2015;15:105.

34. Lin G, Zhao F, Chen H, Deng X, Su J, Zhang T. Comparative phylogeography of the plateau zokor (Eospalax baileyi) and its host-associated flea (Neopsylla paranoma) in the Oinghai-Tibet Plateau. BMC Evol Biol. 2014;14(180):2-9.

35. Rahelinirina S, Léon A, Harstskeerl RA, Sertour N, Ahmed A, Raharimanana C, et al. First isolation and direct evidence for the existence of large small-mammal reservoirs of Leptospira sp. in Madagascar. PloS One. 2010;5(11):e14111.

36. Gardner MG, Cooper SJB, Bull CM, Grant WN. Isolation of microsatellite loci from a social lizard, Egernia stokesii, using a modified enrichment procedure. Hered. 1999;90(2):301-4.

37. Watts PC, Wu JH, Westgarth C, Thompson DJ, Kemp SJ. A panel of microsatellite loci for the southern damselfly, Coenagrion mercuriale (Odonata: Coenagrionidae). Conserv Genet. 2004;5(1):117-9.

38. Rousset F. GENEPOP'007: a complete re-implementation of the GENEPOP software for windows and Linux. Mol Ecol Res. 2008;8:103-6.

39. Weir BS, Cockerham CC. Estimating F-statistics for the analysis of population structure. Evolution. 1984;38(6):1358-70

40. Van Oosterhout C, Hutchinson WF, Wills DPM, Shipley P. MICRO-CHECKER: software for identifying and correcting genotyping errors in microsatellite data. Mol Ecol Notes. 2004:4:535-8.

41. Excoffier L, Lischer HEL. Arlequin suite ver 3.5: a new series of programs to perform population genetics analyses under Linux and windows. Mol Ecol Res. 2010;10(3):564-7.

42. Chapuis M-P, Estoup A. Microsatellite null alleles and estimation of population differentiation. Mol Biol Evol. 2007;24(3):621-31.

43. Pritchard JK, Stephens M, Donnelly P. Inference of population structure using multilocus genotype data. Genetics. 2000;155(2):945-59.

44. Jombart T, Devillard S, Balloux F. Discriminant analysis of principal components: a new method for the analysis of genetically structured populations. BMC Genet. 2010;11(1):94.

45. Earl DA, vonHoldt BM. STRUCTURE HARVESTER: a website and program for visualizing STRUCTURE output and implementing the Evanno method. Conserv Genet Res. 2012:4(2):359-61.

46. Evanno G, Regnaut S, Goudet J. Detecting the number of clusters of individuals using the software STRUCTURE: a simulation study. Mol Ecol. 2005;14(8):2611-20.

47. Jakobsson M, Rosenberg NA. CLUMPP: a cluster matching and permutation program for dealing with label switching and multimodality in analysis of population structure. Bioinformatics. 2007:23(14):1801-6.

48. Rosenberg NA. DISTRUCT: a program for the graphical display of population structure. Mol Ecol Notes. 2004;4(1):137-8.

49. Jombart T. adegenet: a R package for the multivariate analysis of genetic markers. Bioinformatics. 2008;24(11):1403-5.

50. R Development Core Team. R: A language and environment for statistical computing. R Foundation for Statistical Computing. 2008. https://www.rproject.org/. Accessed 2 April 2014.

51. Cornuet JM, Pudlo P, Veyssier J, Dehne-Garcia A, Gautier M, Leblois R, et al. DIYABC v2.0: a software to make approximate Bayesian computation inferences about population history using single nucleotide polymorphism, DNA sequence and microsatellite data. Bioinformatics. 2014;30:1187-9.

52. Rodhain F, Perez C. Précis d'entomologie médicale et vétérinaire. Paris: Maloine; 1985.

53. Kreppel KS, Telfer S, Rajerison M, Morse A, Baylis M. Effect of temperature and relative humidity on the development times and survival of Synopsyllus 
fonquerniei and Xenopsylla cheopis, the flea vectors of plague in Madagascar. Parasit Vectors. 2016;9(82):2-10.

54. Cornuet J-M, Ravigné V, Estoup A. Inference on population history and model checking using DNA sequence and microsatellite data with the software DIYABC (v1. 0). BMC Bioinform. 2010;11(1):401.

55. Cornuet J-M, Santos F, Beaumont MA, Robert CP, Marin J-M, Balding DJ, et al. Inferring population history with DIY ABC: a user-friendly approach to approximate Bayesian computation. Bioinformatics. 2008;24(23):2713-9.

56. Keenan K, McGinnity P, Cross TF, Crozier WW, Prodöhl PA. diveRsity: an R package for the estimation and exploration of population genetics parameters and their associated errors. Met Ecol Evol. 2013;4(8):782-8.

57. Jost $L$. G $G_{S T}$ and its relatives do not measure differentiation. Mol Ecol. 2008;17(18):4015-26.

58. Sundqvist L, Keenan K, Zackrisson M, Prodöhl P, Kleinhans D. Directional genetic differentiation and relative migration. Ecol Evol. 2016:6(11):3461-75.

59. Brouat C, Rahelinirina S, Loiseau A, Rahalison L, Rajerison M, Laffly D, et al. Plague circulation and population genetics of the reservoir Rattus rattus: the influence of topographic relief on the distribution of the disease within the Madagascan focus. PLoS Negl Trop Dis. 2013;7(6):e2266.

60. Fogelqvist J, Niittyvuopio A, Agren J, Savolainen O, Lascoux M. Cryptic population genetic structure: the number of inferred clusters depends on sample size. Mol Ecol Res. 2010;10:314-23.

61. Rosenberg NA, Burke T, Elo K, Feldman MW, Freidlin PJ, Groenen MAM, et al. Empirical evaluation of genetic clustering methods using multilocus genotypes from 20 chicken breeds. Genetics. 2001;159(2):699-713.

62. Tollenaere C, Brouat C, Duplantier J-M, Rahalison L, Rahelinirina S, Pascal M, et al. Phylogeography of the introduced species Rattus rattus in the western Indian Ocean, with special emphasis on the colonization history of Madagascar. J Biogeogr. 2010;37:398-410.

63. Vogler AJ, Chan F, Nottingham R, Andersen G, Drees K, Beckstrom-Sternberg SM, et al. A decade of plague in Mahajanga, Madagascar: insights into the global maritime spread of pandemic plague. MBio. 2013;4(1):e00623.

64. McCoy K. The population genetic structure of vectors and our understanding of disease epidemiology. Parasite. 2008;15:444-8.

65. Herrera F, Urdaneta L, Rivero J, Zoghbi N, Ruiz J, Carrasquel G, et al. Population genetic structure of the dengue mosquito Aedes aegypti in Venezuela. Mem Inst Oswaldo Cruz. 2006:101(6):625-33.

66. Paupy C, Chantha N, Vazeille M, Reynes J-M, Rodhain F, Failloux A-B. Variation over space and time of Aedes aegypti in Phnom Penh (Cambodia): genetic structure and oral susceptibility to a dengue virus. Genet Res. 2003;82(3):171-82.

67. Tien TK, Vazeille-Falcoz M, Mousson L, Hoang TH, Rodhain F, Huong NT, Failloux A-B. Aedes aegypti in ho chi Minh City (Viet Nam): susceptibility to dengue 2 virus and genetic differentiation. Trans R Soc Trop Med Hyg. 1999;93(6):581-6.

\section{Submit your next manuscript to BioMed Central and we will help you at every step:}

- We accept pre-submission inquiries

- Our selector tool helps you to find the most relevant journal

- We provide round the clock customer support

- Convenient online submission

- Thorough peer review

- Inclusion in PubMed and all major indexing services

- Maximum visibility for your research

Submit your manuscript at www.biomedcentral.com/submit
Biomed Central 